\title{
Studie zur globalen Entwick- lung von Wissenschaft und Technik ("Delphi '98")
}

Unter diesem Titel hat Bundesminister Dr. Rüttgers im Februar dieses Jahres die vom Fraunhofer-Institut für Systemtechnik und Innovationsforschung (ISI) durchgeführte neue Delphi-Umfrage vorgestellt. An dieser zweistufigen Delphi-Umfrage haben sich in der 1. Runde 2450 Experten und in der 2. Runde 1866 Experten beteiligt. Kennzeichen einer DelphiBefragung ist, daß den Befragten, die in der 1. Befragungsrunde geantwortet haben, in einer zweiten Befragungsrunde das Meinungsbild der anderen Experten rückgekoppelt wird, so daß der einzelne Experte seine Antwort mit der Einschätzung seiner Kollegen vergleichen und ggf. überdenken kann.

Als Quintessenz der Delphi-Untersuchung hebt Bundesminister Rüttgers in seiner Pressemitteilung vom 17. Februar 1998 folgendes hervor:

- $\quad$ "Die Entwicklungsdynamik in den einzelnen Bereichen ist sehr unterschiedlich. Bei Mobilität und Transport, Information, Dienstleistung und Konsum, Management und Produktion wird das kommende Jahrzehnt die größten Veränderungen bringen. Bei Energie und Rohstoffen sowie Raumfahrt fallen die erwarteten Innovationen mehrheitlich ins zweite und dritte Jahrzehnt des 21. Jahrhunderts.

- Die Experten bestätigen, daß die USA bei Wissenschaft und Technik führend sind. In bestimmten Zukunftsbereichen aber hat Deutschland die Nase vorn:

- Bei Umwelt, Energie, Bauen, Mobilität verfügt Deutschland über eine stärkere FuE-Position als die USA. Japan liegt auf Platz drei.

- Bei Chemie, Produktion, Biomedizin, Raumfahrt und Großexperimenten haben die USA zwar eine Spitzenposition, Deutschland folgt aber dicht auf Position zwei.

- Bei Information und Dienstleistungen führen ebenfalls die USA, gefolgt von Japan. Deutschland steht hier auf dem dritten Platz. 
- Nach Einschätzungen der Fachleute bleibt das Thema Beschäftigung kurz- und mittelfristig eine Achillesferse. Ohne organisatorische Veränderungen in Unternehmen, Flexibilisierung der Beschäftigungszeiten und die Wiedereinführung arbeitsintensiver Dienstleistungen sind positive Beschäftigungswirkungen nicht möglich. An der Kostenfront jedenfalls entscheidet sich das Beschäftigungsthema nicht, so die Delphi-Befragung. Dagegen wird die Bildung und ihre ständige Aktualisierung für den Einzelnen und seinen beruflichen Werdegang immer wichtiger."

Als wichtigste Ergebnisse wertet der Minister folgende:

- $\quad$ Die Unternehmensstrukturen werden sich deutlich verändern: Das Unternehmen der Zukunft wird seine Produkte, Dienstleistungen und inneren Abläufe radikal am Kunden orientieren.

Es wird - stärker als jemals zuvor - auf die Leistungsfähigkeit und Verantwortungsbereitschaft der Mitarbeiter setzen. Der Arbeitnehmer von morgen ist vielfach Mitunternehmer. Das erfordert neue innerbetriebliche Organisationsformen.

Die Beziehungen zwischen Unternehmen werden sich ändern. Stichwort sind vernetzte Unternehmen und Telearbeit. Am Standort Deutschland entstehen Produktionsverbünde mit hoher Reaktionsfähigkeit und Spezialisierung auf Kundengruppen. Insbesondere kleine und mittlere Unternehmen schließen sich zu Verbünden auf Zeit zusammen.

- Multimedia wird zur Alltagstechnik. Die technische Infrastruktur der Wissensgesellschaft wird komplett ausgebaut sein. Die Dynamik im Multimedia-Bereich wird noch größer werden. Im rund um die Uhr geöffneten elektronischen Supermarkt einzukaufen wird genauso zum Alltag gehören wie per Mausklick die Urlaubsreise zu buchen, wobei in beiden Fällen mit digitalem Geld bezahlt wird.

- Es werden neue Weiterbildungssysteme in Beruf und Alltag, also eine neue Lernkultur entstehen. Virtuelle Weltuniversitäten und Volkshochschulen sind weit verbreitet.
Systeme zum Erwerb von MultimediaInformationen "on demand" liegen weltweit in jeder der gängigsten Weltsprachen in Netzwerken dezentral bereit. Sprachübersetzungssysteme im Taschenformat machen grenzenlose Kommunikation möglich.

Die neuen Strukturen vor allem in Fortund Weiterbildung entwickeln sich mit Hilfe der Telekommunikation. Neue Möglichkeiten eröffnen sich für Menschen mittleren und höheren Alters, die mit speziellen Wiederauffrischungs- und Trainingssystemen fit bleiben können. Das "lebenslange Lernen" ist ebenso selbstverständlich wie alltäglich. Es führt vermehrt zu individuellen Qualifikationsbündeln, die mit allgemeingültigen Abschlüssen konkurrieren.

- In der zweiten Dekade des 21. Jahrhunderts sind folgende wichtige Entwicklungen zu erwarten: Für die Zeit nach 2015 zeichnen sich technische Möglichkeiten ab, ökologische Schäden zu reparieren. So wird z.B. die Wiederaufforstung der tropischen Regenwälder für möglich gehalten. Pflanzen sollen wachsen, die eine weitere Ausbreitung der Wüsten verhindern.

- Mit einem Anteil von über $10 \%$ an der Stromerzeugung werden die Erneuerbaren Energien ihren Marktanteil gegenüber heute 20fach vergrößert haben - sogar ohne Einbeziehung der Wasserkraft. Bemerkenswerte Fortschritte wird es bei der Bekämpfung ökologischer Gefahren und zur Verringerung von $\mathrm{CO}_{2}$-Emissionen geben: mit technischen Lösungen im Bereich des Fahrzeugbaus oder im Baubereich."

(Quelle: BMBF-Presse-Info v. 17.2.98)

Es kann hier nicht auf Ergebnisse im einzelnen eingegangen werden. Bundesminister Dr. Jürgen Rüttgers möchte aber Delphi zu einem zentralen Steuerungsinstrument für die mittelund langfristige Innovationspolitik machen. Deshalb sollen weitere Auswertungen des umfangreichen Datenmaterials vorgenommen werden. Zur Verbreitung der Ergebnisse sollen Workshops und Seminare beitragen, auch die Herausgabe eines Delphi-Newsletters ist geplant. Es soll eine Debatte um die Zukunftsentscheidungen in Deutschland angestoßen wer- 
den. Insgesamt werden damit hohe Erwartungen mit dem Delphi-Prozeß verbunden.

Was für die Zukunft interessant sein könnte, wäre eine systematische Analyse der Nutzung der Ergebnisse in der staatlichen und privatwirtschaftlichen Forschungs- und Technologiepolitik. Bei den vorangegangenen Delphi-Erhebungen in Deutschland hat man den Eindruck gewonnen, daß ihre Nutzung eher gering war. In anderen Ländern, z.B. Großbritannien und Schweden, werden DelphiBefragungen eher als ein ergänzendes Instrument im Vorausschauproze $\beta$ gesehen. Der Schwerpunkt wird dort auf Expertenpanel gesetzt, in denen wirkliche Kommunikation zwischen Experten stattfindet und nicht nur vermittelte.

\section{Bibliographische Angaben}

Fraunhofer-Institut für Systemtechnik und Innovationsforschung (Hrsg.): Delphi '98: Studie zur globalen Entwicklung von Wissenschaft und Technik. Zusammenfassung der Ergebnisse. Karlsruhe 1998 Ders.: Delphi '98: Studie zur globalen Entwicklung von Wissenschaft und Technik. Methoden- und Datenband. Karlsruhe 1998.

Die Studie (beide Bände) kann für DM 55,-- (zzgl. Versand und MwSt) bezogen werden beim Fraunhofer-Institut für Systemtechnik und Innovationsforschung, Breslauer Str. 48, D-76139 Karlsruhe, Fax: +49 (0) $721 / 689152$. 